\title{
Information on the Food Security Situation and Perspective
}

\author{
Siloyde Rivas* \\ Department of Health and Nutrition Consultant, Venezuela \\ *Corresponding Author: Siloyde Rivas, Department of Health and Nutrition Consultant, Venezuela.
}

Received: September 16, 2019; Published: November 01, 2019

DOI: $10.31080 /$ ASNH.2019.03.0518

The concept of food security responds to the satisfaction of the right to food as a fundamental human right, seeks strategic autonomous supply, not autocrachial, it is inserted in the comprehensive development scheme itis projected in a world of global markets, considers the availability of food as an agronomic and international trade problem and promotes accessibility. Identifying risk factors by considering food security as a matter of rights facilitates the definition of actions to protect the most vulnerable families, within which, in addition to the poorest households, the smallest unorganized producers. However, national average figures do not show the inequalities present on the continent, especially in peripheral and rural urban areas.

The global economic crisis has generated negative economic growth figures for the region, which implies declining productivity, declining employment figures, with the wage rate being the most affected, in which a predominant role is assigned to the agricultural sector. Rising prices have an on record of lower revenue, impaired purchasing power and loss of sources of income. As a result, progress in the increase in food-safe households is stalled, at the risk of regressing if the necessary measures are not taken. The impact of the situation is seen as depending on the degree of transfer of the global crisis to local markets, especially food.

Existing food security policies need to be known and evaluated in order to identify gaps and to establish coherent and feasible proposals to implement. These policies should be implemented as short-, medium- and long-term state policies.

Some measures taken relate to programmes for the control of temporary prices of basic foodstuffs, subsidies for these foods and direct distribution of food to vulnerable groups. However, it should be emphasized that productivity has improved as a source of stable employment, a strengthening action of the right to food security.

On the other hand, there is a common denominator in the identified problem, both at the regional level, at the national level and at the local level: the absence of information, that supports decision-making or the design of plans and programmes related to the issue of food security.
Food security information system required, especially early warning subsystem that identifies vulnerabilities and anticipates insecurity situations derived from its two key components, availability and access to food, in order to prevent deterioration of nutritional status, especially in the most vulnerable groups of the population: maternal children and older adults.

The purpose of providing solutions is, with reference to information systems, to use existing state-of-the-art technology, interconnected with all levels of information generation, which allows the systematization and geo reference of indicators necessary for decision-making.

The main dynamize ring elements would be represented by information generators and users, accompanied by institutional actors responsible for technology platforms and academic support of universities.

The importance of educational programmes in community nutrition is mentioned as a contribution to improving food security in communities, when they are part of agri-food development programmes.

The actors responsible for energizing these processes would be represented by programme and service managers, in the public sector and private, in the different areas of action aimed at the sustainability of security Food.

\section{Volume 3 Issue 12 December 2019 (C) All rights are reserved by Siloyde Rivas.}

